\title{
On the endosomal function and gene nomenclature of human SPE-39
}

\section{To the Editor:}

It was a great satisfaction to see our findings on the endosomal function of SPE-39, a Vps33b-interacting protein, recapitulated in a recent study by Cullinane et al. ${ }^{1}$, as we previously identified a phylogenetically conserved function for SPE-39 in the endocytic route ${ }^{2,3}$. However, we think that the journal missed an important opportunity to respect existing gene nomenclature and failed to record the availability of our published antibody resource.

In a 2003 study $^{2}$, we discovered Caenorhabditis elegans spe-39, described the phenotypic consequences of spe-39 loss of function and showed that it had human and Drosophila orthologs, C14ORF133 (FLJ12707) and CG18112. Further work in Drosophila suggested that there was an interaction between Vps33b and SPE-39 orthologs ${ }^{4,5}$, but the functional importance of this interaction was not determined. Subsequently, we defined the function of the human C14ORF133 gene product ${ }^{3}$. First, we showed that human and C. elegans SPE-39 interact with Vps33b and form a complex with the hexameric HOPS complex ${ }^{3}$. Second, we showed that human SPE-39 (C14ORF133) is required for trafficking from early stages of the endocytic pathway
(Rab11 and Rab5 compartments) toward lysosomes both in human and in several cell types in C. elegans ${ }^{3}$.

The paper you published ${ }^{1}$ stated "As no anti-VIPAR antibody was available, we used transfections of epitope-tagged.... This statement was incorrect as published because we had already produced a monoclonal antibody against residues 406-493 of human SPE-39 (the conceptual translation product of C14ORF133) and it was used for experiments described in our paper ${ }^{3}$. We have made this antibody available to the scientific community according to US National Institutes of Health and editorial guidelines. The use of this antibody enables experiments that detect endogenous proteins without the need for transgenic constructs that may not faithfully reproduce physiological levels of expression.

Finally, we believe that renaming C14ORF133 as "VIPAR" makes it difficult to follow the relationships between orthologous genes as well as the chain of published evidence for the function of the human gene and its product. Studies in model organisms have contributed many names for new genes that have been kept when orthologs were later discovered in humans. For example, Saccharomyces cerevisiae mutants that affect vacuolar assembly were named vps mutants to indicate defective vacuolar protein sorting ${ }^{6}$. Later identified orthologs of yeast vps33 in Drosophila, mouse and human adopted the yeast name. We therefore favor the use of human SPE-39 as standard nomenclature. This name change would reestablish the historically and scientifically justified connection of SPE39 (C14ORF133) to the research articles in which it was first described.

\section{COMPETING FINANCIAL INTERESTS}

The authors declare no competing financial interests.

Steven W L'Hernault ${ }^{1}$ \& Victor Faunde $z^{2}$

${ }^{1}$ Department of Biology, Emory University, Atlanta, Georgia, USA. ${ }^{2}$ Department of Cell Biology, Emory University, Atlanta, Georgia, USA. Correspondence should be addressed to S.W.L'H. (bioslh@emory.edu) orV.F. (faundez@cellbio.emory.edu).

1. Cullinane, A.R. et al. Nat. Genet. 42, 303-312 (2010).

2. Zhu, G.D. \& L'Hernault, S.W. Genetics $165,145-157$ (2003).

3. Zhu, G.D. et al. Mol. Biol. Cell 20, 1223-1240 (2009).

4. Pulipparacharuvil, S. et al. J. Cell Sci. 118, 36633673 (2005)

5. Giot, L. et al. Science 302, 1727-1736 (2003).

6. Rothman, J.H., Yamashiro, C.T., Kane, P.M. \& Stevens, T.H. Trends Biochem. Sci. 14, 347-350 (1989). 